$$
\begin{array}{llllll} 
& \mathfrak{l} & \mathfrak{h} & \mathfrak{a} & \mathfrak{l} & \mathbf{t}
\end{array}
$$

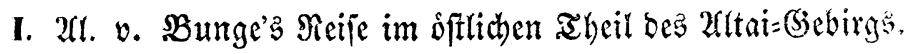

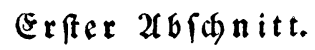

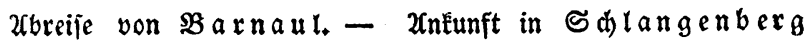
und 2fufentyalt bajerbft. - Reife nact) bem Dorfe $\mathfrak{z}$ [d et $t=$

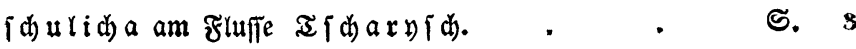
$3 \mathfrak{w e i t e r} 2 \mathfrak{B}\{d \mathfrak{n i t}$.

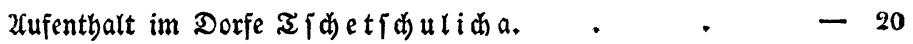

Dritter $\mathfrak{A} \mathfrak{b}$ d $\mathfrak{n}$ it t.

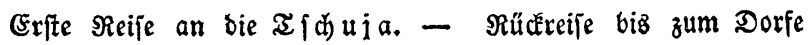
uimon.

\title{
Dierter $\mathfrak{A} \mathfrak{b}$ id $\mathfrak{n}$ itt.
}

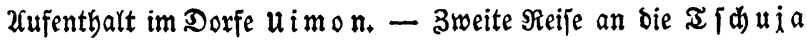
uno zum telez genberg.

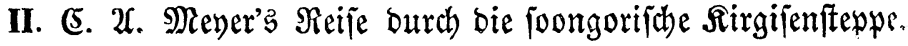

$$
\text { Erefter } \mathfrak{A} \mathfrak{b}\{\mathfrak{d} \mathfrak{n} \mathfrak{i t} \text { t. }
$$

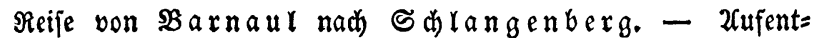

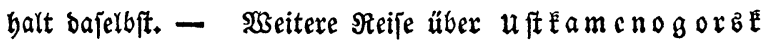

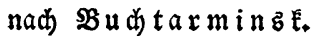

Zweiter $2(\mathfrak{b}$ (d) $\mathfrak{n}$ itt.

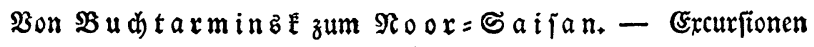

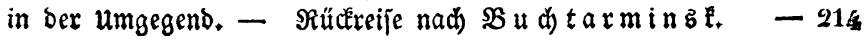

Dritter 20 fditt.

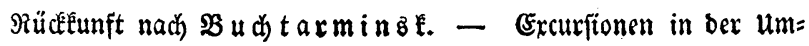
gegend. 


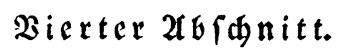

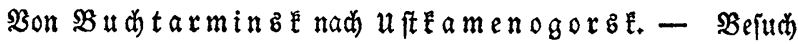
ber Ruinen von $2 \mathfrak{b} \mathfrak{l} a \mathfrak{k}$ t. - Reife nad) Semipala= tinz $\mathfrak{E}_{+}-\mathcal{U}$ Uffenthalt bajelbit.

ธ. 319

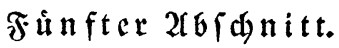

Reije burdy bie Rirgijenfteppe nady $\mathfrak{I} \mathfrak{a} \mathfrak{r}=\mathfrak{R} \mathfrak{a} \mathfrak{r} \mathfrak{a} \mathfrak{y}$.

Sedster $2 \mathfrak{b}$ \{dnitt.

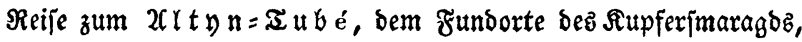

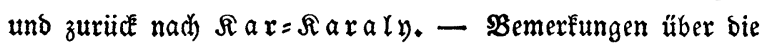
Nieberlaffung in $\mathfrak{A} \mathfrak{a} \mathfrak{r}=\mathfrak{K} \mathfrak{a} \mathfrak{a} \mathfrak{l} \mathfrak{y}$. - Semerkungen über die Ringifen.

\section{Siebenter Ub}

Rücéreije nad Semipalatinz

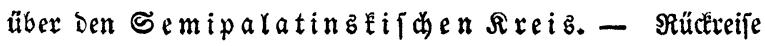
$\mathfrak{n a d}) \mathfrak{B} \mathfrak{a} \mathfrak{i} \mathfrak{a} \mathfrak{u} \mathfrak{l} . \quad$. . . . .

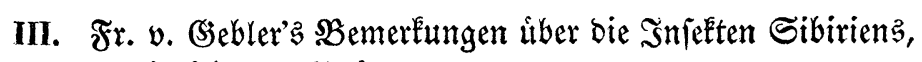
vorzúglich bes 2ultai. 